\title{
Differential expression patterns of GATA3 in usual and differentiated types of vulvar intraepithelial neoplasia: potential diagnostic implications
}

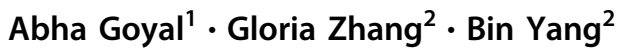 \\ Received: 31 October 2017 / Revised: 20 December 2017 / Accepted: 21 December 2017 \\ (c) United States \& Canadian Academy of Pathology 2018
}

\begin{abstract}
The two main precursors of vulvar squamous cell carcinoma, usual and differentiated vulvar intraepithelial neoplasia (VIN), have distinctive etiology, pathogenesis, and natural history. Usual type VIN is often associated with high-risk HPV and differentiated VIN has de novo p53 genetic alterations that are unrelated to HPV infection. GATA-binding protein 3 (GATA3) is a tumor suppressor that shows increased expression in several types of human malignancies including breast and bladder carcinomas. Little is known regarding the expression of GATA3 in vulvar squamous neoplasms. We have systematically examined the expression of GATA3 in 119 vulvar lesions and neoplasms including 20 cases of lichen sclerosus, 12 cases of lichen simplex chronicus, 30 cases of usual type VIN, 34 cases of differentiated VIN, and 23 cases of squamous cell carcinoma. Similar to adjacent non-neoplastic epidermis, moderate to strong GATA3 expression was retained in all cases of lichen sclerosus, lichen simplex chronicus, and usual type VIN. However, in comparison, the GATA3 immunostaining pattern in differentiated VIN was distinct. Partial/complete loss of GATA3 expression in the basal layer with or without loss in the parabasal layer was observed in 30/34 (88\%) of differentiated VIN cases. Significant loss of GATA3 expression was also observed in all (7/7) squamous cell carcinomas associated with usual type VIN and in 13/16 $(81 \%)$ of those associated with differentiated VIN. There was no significant correlation between loss of GATA3 expression and overexpression of p53 in differentiated VIN. Our study shows that loss of GATA3 expression is seen in the vast majority $(87 \%)$ of vulvar squamous cell carcinomas. Downregulation of GATA3 may be an early event during tumorigenesis in differentiated VIN but not in HPV-related usual type VIN. Our data suggests that application of GATA3 immunohistochemistry along with p53 may be a useful tool in facilitating the accurate diagnosis of VIN.
\end{abstract}

\section{Introduction}

Squamous cell carcinoma constitutes $\sim 95 \%$ of the malignant neoplasms of the vulva [1]. The precursor lesion, i.e., vulvar intraepithelial neoplasia (VIN) is categorized mainly into two types-the usual type and the differentiated type. The two types of VIN are distinct from each other in terms of their epidemiology, etiology, clinical presentation,

Bin Yang

yangb@ccf.org

1 Weill Cornell Medicine, New York Presbyterian Hospital, 525 East 68th Street, F766, New York, NY 10065, USA

2 Department of Pathology, Robert J. Tomsich Pathology and Laboratory Medicine Institute, Cleveland Clinic, 9500 Euclid Avenue, L25, Cleveland, OH 44195, USA pathologic features, and natural history. Usual type VIN is associated with high-risk human papilloma virus (HPV) infection, presents at a relatively younger age, has readily recognizable pathologic features (mostly a combination of basaloid and warty morphologies), and has a low risk of progression to invasive carcinoma. Differentiated VIN, on the other hand, is a considerably different entity. It is often seen in association with chronic dermatoses such as lichen sclerosus, is typically independent of HPV, presents at an older age, has subtle pathologic features, and has a high risk of progression with a propensity for early invasion [2-4]. In contrast to usual type VIN where $5.7 \%$ progress to invasive carcinoma, 33\% of differentiated VIN will develop invasion [5]. Though majority of the VINs are HPV-related, most of the invasive carcinomas are not. Recent studies have shown that while HPV DNA can be detected in $80.4-86.7 \%$ of VIN, it is found only in $28.6-40.1 \%$ of invasive vulvar carcinomas [6-8]. Also, this progression to invasive 
carcinoma happens earlier in differentiated VIN as compared to usual type VIN (22.8 months versus 41.4 months) [5].

The aggressive biologic behavior of differentiated VIN is compounded by the difficulties in its clinical and pathologic recognition [4]. On light microscopy on hematoxylin and eosin (H\&E) stain, differentiated VIN is a challenging diagnosis due to its highly mature appearance that is accompanied by lack of obvious architectural disarray and diffuse nuclear atypia [2]. Furthermore, the etiology and pathogenesis of this disease is not completely understood with most studies focusing on the frequently detected p53 mutations [9-12]. There is a need for biomarkers that can assist in the diagnosis of differentiated VIN and perhaps aid in the identification of molecular mechanisms involved in it.

GATA-binding protein 3 (GATA3) is a transcription factor with zinc fingers of $\mathrm{C} 2 \mathrm{H} 2$ type that promotes cellular differentiation. These nuclear proteins recognize A/T G-AT-A A/G nucleotide sequences in gene promoters and activate or repress those genes. It has an important role in the development of $\mathrm{T}$ cells, mammary gland luminal epithelium, skin, inner ear, nervous system, and kidney [13]. More specifically, in the skin, GATA3 plays a role in the regulation of the differentiation of the inner root sheath in hair follicles and has been shown to inhibit proliferation and induce differentiation of primary keratinocytes [14]. Although a few studies have shown that GATA3 is highly expressed in squamous cell carcinoma of the skin but less so in those of the cervix and lung, little is known regarding the expression patterns of GATA3 in VIN and in vulvar squamous cell carcinoma $[15,16]$. The aim of this study was to assess the immunohistochemical expression of GATA3 in non-neoplastic epidermis, lichen sclerosus, lichen simplex chronicus, VIN including both usual and differentiated types, and invasive vulvar squamous cell carcinoma.

\section{Materials and Methods}

This study was approved by the Institutional Review Board at the Cleveland Clinic. A retrospective database search from the pathology laboratory information system was performed between 7 January 2005 and 30 June 2014. A total of 119 cases-including 20 cases of lichen sclerosus with adjacent normal skin, 12 cases of lichen simplex chronicus, 30 cases of usual type VIN, 34 cases of differentiated VIN, and 23 cases of invasive vulvar squamous cell carcinoma with available tissue blocks were included in this study. The H\&E slides were reviewed to confirm the histologic diagnosis and the most representative block was chosen for immunohistochemistry. Prior to the performance of immunohistochemistry, a recut slide was stained with
$\mathrm{H} \& \mathrm{E}$ to confirm the presence of the lesion or the tumor. GATA3 immunohistochemistry was performed on all study cases, p53 immunohistochemistry only on differentiated VIN cases and p16 immunohistochemistry only on usual type VIN cases.

The following antibodies were employed: anti-GATA3 antibody (L2-823 clone, mouse monoclonal, Biocare, Concord, CA), anti-p53 antibody (Do-7 clone, mouse monoclonal, Dako, Carpenteria, CA), and anti-p16 antibody (E6H6 clone, mouse monoclonal, Ventana, Tucson, AZ). Epitope retrieval was performed using high pH EDTA retrieval buffer for $32 \mathrm{~min}$. The antibody incubation time at room temperature was $24 \mathrm{~min}$. The immunohistochemical staining was detected using the Ventana Medical system benchmark ultra biotin-streptavidin-binding DAB detection kit. Positive and negative controls were included with each run. Only nuclear labeling was considered as positive staining for both GATA3 and p53 immunohistochemistry. Positive staining was recorded in terms of: epithelial distribution (e.g., basal layer versus parabasal layer), percentage of positive cells per 100 consecutive cells (0-25, $26-50,51-75 \%$, or $>75 \%$ ) and intensity of staining (weak 1 + , moderate $2+$, or strong $3+$ ). For p16 immunohistochemistry, block positivity was interpreted as positive staining, i.e., strong nuclear and cytoplasmic staining in a continuous distribution involving at least lower half of the epithelium.

\section{Results}

\section{Expression of GATA3 in histologically normal epidermis, lichen sclerosus, and lichen simplex chronicus}

Histologically normal epidermis was identified in 45 cases adjacent to the lesion of interest, i.e., lichen sclerosus in 20 cases and VIN in 25 cases. GATA3 immunostaining was observed in all keratinocytes from the basal layer to the spinous layer. Moderate staining intensity $(2+)$ was observed in $32(71 \%)$ cases and strong staining intensity (3 $+)$ in $13(29 \%)$ cases. The granular cell layer revealed few nuclei with minimal weak staining and the stratum corneum did not show any staining (Fig. 1a, b).

Twenty cases of lichen sclerous (all biopsies) without any atypia and with no prior history of VIN and/or vulvar squamous cell carcinoma showed a GATA3 staining pattern similar to that of the normal epidermis. Moderate staining intensity $(2+)$ was observed in $4(20 \%)$ cases and strong intensity (3+) in $16(80 \%)$ cases (Fig. 1c, d).

Twelve cases of vulvar lichen simplex chronicus (all biopsies) was also included in this study. None of these were associated with squamous dysplasia or malignancy. 

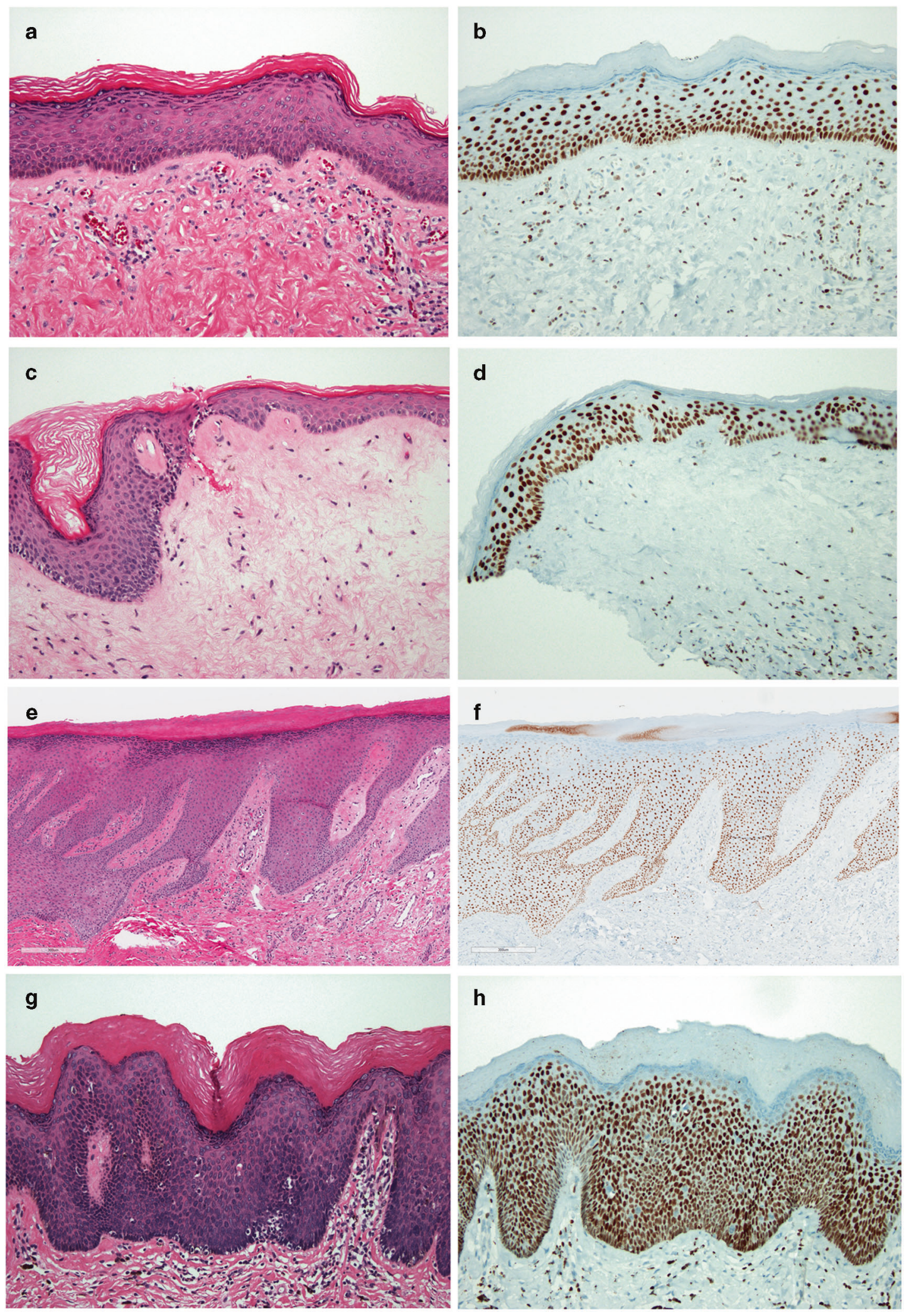

Fig. 1 GATA3 immunohistochemical staining patterns in normal epidermis, lichen sclerosus, lichen simplex chronicus and usual type VIN. Normal epidermis $(\mathbf{a}, \mathbf{b})(\times 200)$ revealed moderate to strong staining from the basal to the spinous layers of the epidermis. Only

rare nuclei exhibited weak staining in the granular cell layer. Lichen sclerosus $(\mathbf{c}, \mathbf{d})(\times 200)$, lichen simplex chronicus $(\mathbf{e}, \mathbf{f})(\times 200)$, and usual type VIN $(\mathbf{g}, \mathbf{h})(\times 200)$ showed staining patterns similar to that of non-neoplastic epidermis 

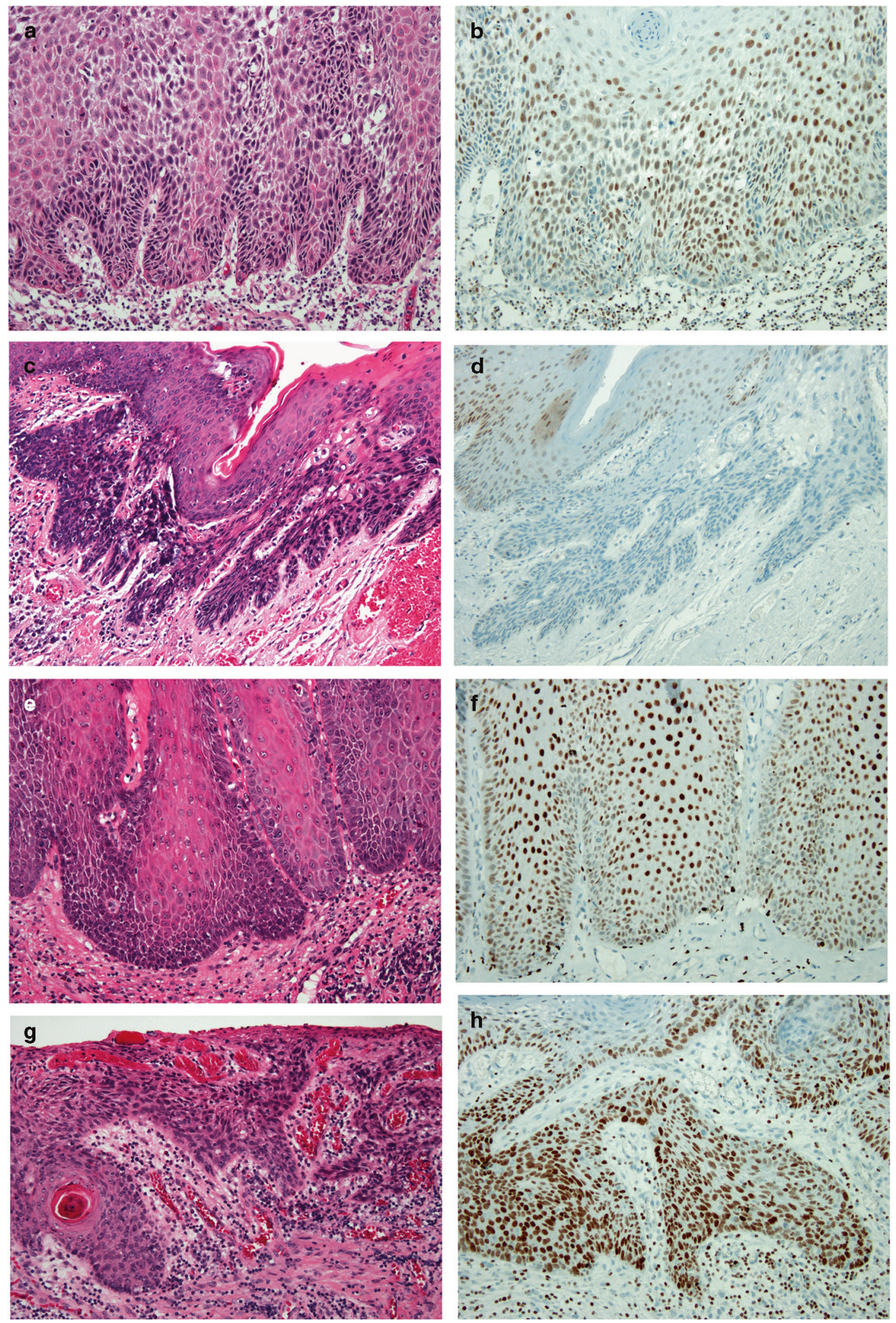

Fig. 2 GATA3 immunohistochemical staining patterns in differentiated VIN. Case 1 a $(H \& E, \times 200)$ shows prominent loss of GATA3 staining in the basal cell layer b $(\times 200)$. Case 2 c $(H \& E$, $\times 200$ ) shows striking loss of staining in the parabasal layers as well $\mathbf{d}$

$(\times 200)$. Case 3 e $(H \& E, \times 200)$ shows less obvious loss of staining in the basal cell layer $(0-1+$ staining in $<50 \%$ of basal cells $) \mathbf{f}(\times 200)$. Case $4 \mathbf{g}(\mathrm{H} \& \mathrm{E}, \times 200)$ reveals no appreciable loss of staining in the epidermis $\mathbf{h}(\times 200)$ 
The staining pattern was comparable to that of normal epidermis and of lichen sclerosus. Moderate staining intensity $(2+)$ was observed in $1(8 \%)$ case and strong intensity $(3+)$ in $11(92 \%)$ cases (Fig. 1e, f).

\section{Expression of GATA3 in usual type VIN and differentiated VIN}

All 30 cases of usual type VIN, including 15 excisions and 15 biopsies, revealed intact GATA3 staining in the keratinocytes. Moderate staining intensity $(2+)$ was observed in 9 $(25 \%)$ cases of usual type VIN and strong intensity $(3+)$ in $27(75 \%)$ cases (Fig. 1g, h). Overall, GATA3 staining in usual type VIN was similar in intensity or slightly stronger as compared to the adjacent non-neoplastic epidermis. All 30 cases of usual type VIN were confirmed on p16 immunohistochemistry with a block staining pattern in the lesional cells.

The pattern of GATA3 expression was distinct in differentiated VIN. Based on the staining distribution and the percentage of positive cells, GATA3 immunoreactivity in differentiated VIN was categorized into three immunostaining patterns: 0,1 , and 2 . Pattern 0 indicates a staining pattern in the lesional epithelium that was similar to that of the adjacent non-neoplastic vulvar epidermis. Pattern 1 refers to the loss of GATA3 immunoreactivity in 25-75\% cells of the basal layer of differentiated VIN. Pattern 2 refers to the loss of GATA3 immunoreactivity in $>75 \%$ cells of the basal layer that was accompanied by a prominent decrease in GATA3 staining (either weak staining or complete lack of) in the parabasal layer as well. Overall, near-complete or partial loss of GATA3 expression was seen in the vast majority 30/34 (88\%) of differentiated VIN cases-pattern 1 in 7 (20\%) cases and pattern 2 in $23(68 \%)$ cases. Pattern 0 with retained strong GATA3 immunoreactivity was seen in the remaining 4 (12\%) cases (Table 1) (Fig. 2).

Table 1 Patterns of GATA3 immunohistochemistry in usual type and differentiated VIN

\begin{tabular}{lll}
\hline GATA3 staining pattern & Usual type VIN & Differentiated VIN \\
\hline Pattern 0 & 30 & 4 \\
Pattern 1 & 0 & 7 \\
Pattern 2 & 0 & 23 \\
Total number of cases & 30 & 34 \\
\hline
\end{tabular}

Pattern 0 refers to no significant difference of GATA3 staining of VIN and of adjacent non-neoplastic epidermis; pattern 1 refers to the loss $25-75 \%$ of GATA3 immunoreactivity in the basal layer; pattern 2 refers to the loss of $>75 \%$ of GATA3 immunoreactivity in the basal layer with reduced GATA3 staining in the parabasal layer
Table 2 GATA3 immunohistochemistry in invasive squamous cell carcinomas of vulva

\begin{tabular}{lll}
\hline Type of VIN & $\begin{array}{l}\text { Percentage of tumor cells } \\
\text { with loss of expression }\end{array}$ & $\begin{array}{l}\text { Number of } \\
\text { cases (\%) }\end{array}$ \\
\hline Differentiated type & $>75 \%$ & $13(81.3)$ \\
& $26-50 \%$ & $3(19.7)$ \\
Usual type & $>75 \%$ & $7(100)$ \\
\hline
\end{tabular}

\section{Expression of GATA3 in invasive vulvar squamous cell carcinomas}

Twenty-three cases of invasive squamous cell carcinoma of vulva were included in the study-16 cases associated with differentiated VIN and 7 cases associated with usual type VIN. All 16 invasive carcinomas associated with differentiated VIN showed loss of GATA3 expression in tumor cells-loss in $>75 \%$ tumor cells in $13 / 16(81 \%)$ cases and loss in $26-75 \%$ tumor cells in 3/16 (19\%) cases. All seven invasive carcinomas associated with usual type VIN exhibited loss of GATA3 expression as well-loss in $>75 \%$ tumor cells in $7 / 7$ (100\%) cases (Table 2) (Fig. 3a-d). In those cases with significantly retained GATA3 expression, the staining intensity was relatively weak $(1+)$ in comparison to the non-neoplastic vulvar epidermis and usual type VIN $(2-3+)$.

\section{Correlation of GATA3 and p53 immunohistochemistry patterns in differentiated VIN}

Based on the epithelial distribution, the number of cells staining and the intensity of staining, the p53 immunohistochemical expression in differentiated VIN was categorized into three patterns: 0,1 , and 2. Pattern 0 indicates a p53 staining pattern in differentiated VIN that was not significantly different from the adjacent non-neoplastic vulvar epidermis. Pattern 1 refers to significantly increased p53 labeling in the basal layer with no prominent suprabasal extension. Pattern 2 refers to significantly increased p53 labeling in the basal layer that was accompanied with prominent suprabasal extension. Overall, increased p53 labeling was seen in 29/34 (85\%) of differentiated VIN casespattern 1 in $12(35 \%)$ cases and pattern 2 in $17(50 \%)$ cases. The remaining 5 (15\%) cases of differentiated VIN showed pattern 0 with p53 immunoreactivity that was not significantly different from that of the adjacent non-neoplastic epidermis.

As presented in Table 3, 28/34 (82\%) of differentiated VIN cases had concurrent partial/near-complete loss of GATA3 expression and aberrant expression of p53. Interestingly, of three cases of differentiated VIN with no increase in p53 labeling, two cases showed significant 

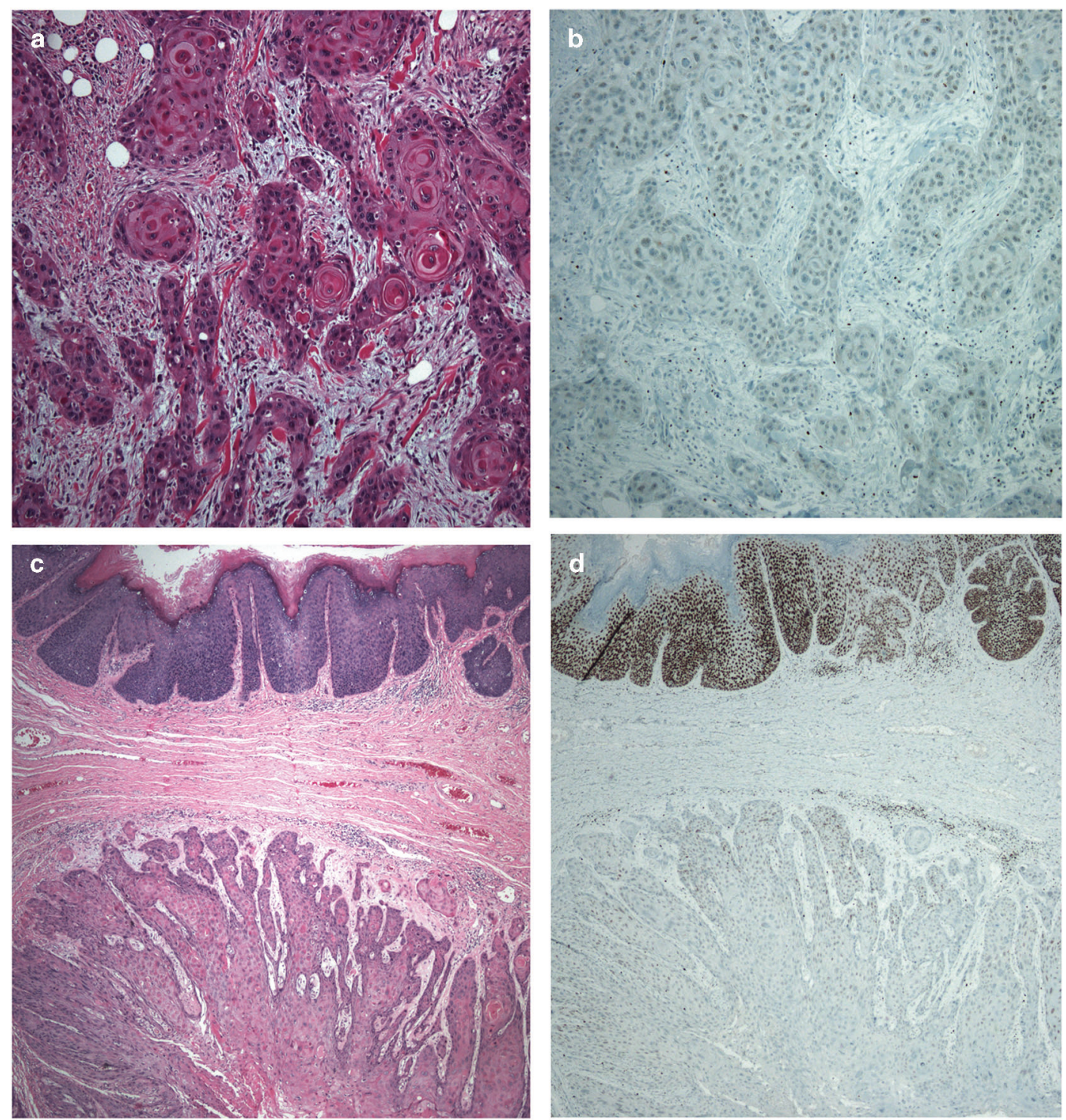

Fig. 3 GATA3 immunohistochemical staining patterns in vulvar invasive squamous cell carcinomas. Invasive squamous cell carcinoma arising in association with differentiated VIN a (H\&E, $\times 200)$ shows

decrease in GATA3 staining (one case of pattern 1 and one case of pattern 2). Conversely, increase in p53 labeling was seen in three differentiated VIN cases that retained strong GATA3 expression (pattern 0 of GATA3 staining). Only one differentiated VIN case had staining pattern 0 for both GATA3 and p53.

\section{Discussion}

Differentiated VIN was initially described by Abell in the 1960s as an "intraepithelial carcinoma of simplex type." It was considered as a histologically subtle lesion as the neoplastic cells were "well differentiated" and were not

marked loss of staining in the tumor cell nuclei $\mathbf{b}(\times 200)$. Invasive carcinoma arising from usual type VIN $\mathbf{c}(H \& E, \times 200)$ shows similar loss of staining $\mathbf{d}(\times 200)$

present throughout the entire thickness of the epithelium [17]. Till date, the diagnosis of differentiated VIN remains difficult and is diagnosed as a solitary lesion only in limited instances, possibly due to its under-recognition or its short intraepithelial phase. The low end of the morphologic spectrum of differentiated VIN is even more problematic to characterize. Van de Nieuwenhof reported that $42 \%$ of lichen sclerosus cases that progressed to vulvar squamous cell carcinoma were reclassified as differentiated VIN on retrospective review [18]. Not surprisingly, the pathologic diagnosis of differentiated VIN suffers from low interobserver reproducibility [19].

Given the difficulties in the histologic identification of differentiated VIN, a variety of immunohistochemical 
Table 3 Correlation between GATA3 and p53 immunostaining patterns in differentiated VIN

\begin{tabular}{|c|c|c|}
\hline Case no. & GATA3 staining pattern & p53 staining pattern \\
\hline 1 & 0 & 0 \\
\hline 2 & 0 & 1 \\
\hline 3 & 0 & 2 \\
\hline 4 & 0 & 2 \\
\hline 5 & 1 & 0 \\
\hline 6 & 1 & 1 \\
\hline 7 & 1 & 1 \\
\hline 8 & 1 & 2 \\
\hline 9 & 1 & 2 \\
\hline 10 & 1 & 2 \\
\hline 11 & 1 & 2 \\
\hline 12 & 2 & 0 \\
\hline 13 & 2 & 1 \\
\hline 14 & 2 & 1 \\
\hline 15 & 2 & 1 \\
\hline 16 & 2 & 1 \\
\hline 17 & 2 & 1 \\
\hline 18 & 2 & 1 \\
\hline 19 & 2 & 1 \\
\hline 20 & 2 & 1 \\
\hline 21 & 2 & 1 \\
\hline 22 & 2 & 2 \\
\hline 23 & 2 & 2 \\
\hline 24 & 2 & 2 \\
\hline 25 & 2 & 2 \\
\hline 26 & 2 & 2 \\
\hline 27 & 2 & 2 \\
\hline 28 & 2 & 2 \\
\hline 29 & 2 & 2 \\
\hline 30 & 2 & 2 \\
\hline 31 & 2 & 2 \\
\hline 32 & 2 & 2 \\
\hline 33 & 2 & 2 \\
\hline 34 & 2 & 2 \\
\hline
\end{tabular}

Please refer to GATA3 and p53 immunostaining patterns in the Results section

markers have been reported to be helpful to varying extents in aiding this diagnosis. One of the most well-studied markers in this regard is p53. Several studies have revealed that p53 mutation is a frequent finding in differentiated VIN and in HPV-negative vulvar squamous cell carcinoma [912]. Most of these are missense mutations, although nonsense mutations have also been reported [10]. Yang and Hart [20] reported in their study that 10 of 12 cases of simplex VIN demonstrated strong, diffuse p53 immunostaining of the basilar cells that was accompanied by suprabasilar extension of the p53 positive cells. Although this pattern of p53 immunostaining can prove valuable in diagnosing differentiated VIN, it is not without limitations. Increased p53 immunostaining has also been reported in lichen sclerosus and in squamous hyperplasia and is possibly due to oxidative stress that causes increased stabilization of the wild-type p53 protein [21, 22]. Differentiated VIN with p53 nonsense mutation shows a null pattern on immunostaining (complete lack of staining of lesional cells) that may be problematic to interpret in small biopsies [23]. Also not all cases of differentiated VIN harbor p53 mutations and will not exhibit the p53 immunostaining patterns that assist in its diagnosis.

GATA3 is a transcription factor critical for embryogenesis, development, and cell differentiation. The role of GATA3 in the progression of breast cancer has been well studied. In mouse models, it has been demonstrated that there is downregulation of GATA3 in advanced mammary carcinoma [24-26]. GATA3 is not a conventional tumor suppressor. It promotes cellular differentiation and its loss or reduced expression supports malignant transformation due to the inability of the cells to mature and exit the cell cycle [13]. It is also highly expressed in urothelial carcinomas and its loss has been associated with worse prognosis. There is limited information regarding the expression of GATA3 in squamous cell carcinomas. In a study by Miettinen et al. [15], GATA3 was reported to be positive in $81 \%$ cutaneous, $33 \%$ cervical, and $16 \%$ laryngeal squamous cell carcinomas. Similarly, Mertens et al. [16] reported that GATA3 was expressed in 21/24 (88\%) of squamous cell carcinomas of skin. Recently, Chang et al. [27] reported that focal and weak GATA3 staining was seen in $6 \%$ anal squamous cell carcinomas and $19 \%$ uterine squamous cell carcinomas, but in none of the 25 pulmonary squamous cell carcinomas. Clark et al. [28] reported GATA3 positivity in three of four vulvar squamous cell carcinomas included in their study. However, to our knowledge, the expression of GATA3 in VIN has not been reported in English literature so far. In this study, we have systematically investigated the immunohistochemical expression of GATA3 in normal vulvar skin, lichen sclerosus, lichen simplex chronicus, differentiated VIN, usual type VIN, and invasive vulvar squamous cell carcinomas.

Our study showed that GATA3 is immunohistochemically expressed in normal vulvar epidermis, mostly with a strong intensity. Such an expression was retained in all cases of lichen sclerosus and lichen simplex chronicus. Expression of GATA3 varied in VIN and was dependent on the type of VIN. In usual type VIN associated with highrisk HPV infection, evidenced by strong block positivity with p16 in our study, the dysplastic epithelium retained moderate to strong GATA3 immunostaining that was not significantly different from that of the adjacent nonneoplastic vulvar epidermis. However, the pattern of 
GATA3 immunostaining in differentiated VIN was distinctive as compared to that in usual type VIN, lichen sclerosus, and lichen simplex chronicus. We found that $\sim 88 \%$ of differentiated VIN cases exhibited loss of GATA3 immunoreactivity, partially or almost completely, in the basal layer of the neoplastic epithelium. In $68 \%$ of differentiated VIN cases, the loss of GATA3 staining was limited not only to the basal layer but it extended to the parabasal layer as well. It is likely that the basal layer of the vulvar epidermis is the initial locus of malignant transformation in differentiated VIN since the overexpression of p53 (presumably correlating with underlying p53 missense mutations) also starts in the basal layer. Downregulation of GATA3 expression, starting in the basal layer, may result in an increase in cell proliferation and in altered cell differentiation. The loss of GATA3 expression in differentiated VIN also suggests that it may be an early molecular event during the tumorigenesis of non HPV-related VIN. A genomic study using GATA3 knockdown keratinocytes in vitro demonstrated that GATA3 is a master regulator of the transcriptional response to low-dose ionizing radiation in human keratinocytes [29]. There is limited literature regarding the relationship between GATA3 and p53 in malignant transformation. Recently, Nakayama et al. [30] reported in an abstract that p53 mutation and GATA3 knockout had synergetic effect in driving malignant transformation in cultured breast cancer cells. Our observation of simultaneous loss of GATA3 expression and overexpression of p53 in the majority of differentiated VIN cases seems to echo their findings of synergetic effect between p53 and GATA3-pRb pathways in breast cancer cells. Though based on our study results we cannot delineate the underlying molecular mechanisms of differentiated VIN in detail, early downregulation of GATA3 along with p53 mutations may contribute to its high oncogenic potential.

As mentioned earlier, p53 alteration is a hallmark of an another early event in non HPV-related carcinogenesis. We have studied the correlation between loss of GATA3 expression and overexpression of p53 in differentiated VIN. We found that concurrent loss of GATA 3 and overexpression of p53 was observed in 28/34 (82\%) of differentiated VIN cases. There were four differentiated VIN cases with retained GATA3 expression (pattern 0) and three cases with no significant p53 overexpression (pattern 0). However, only one differentiated VIN case had expression pattern 0 for both GATA3 and p53. Our data indicates that although the immunohistochemical staining patterns of neither GATA3 nor p53 are perfect for the diagnosis of differentiated VIN, when applied in conjunction, they are informative in the vast majority of cases (33/34 cases in our study). Therefore, employing immunohistochemistry for both p53 and GATA3 may be helpful in aiding the histologic diagnosis of differentiated VIN and in its distinction from usual type VIN.

Another interesting finding of our study was that the vast majority of vulvar squamous cell carcinomas demonstrated significant loss of GATA3 expression, regardless of etiology and the type of VIN with which they were associated. In 16 cases of invasive squamous cell carcinoma associated with differentiated VIN, $81 \%$ cases showed marked loss of GATA3 expression. GATA3 expression was significantly retained in three cases of squamous cell carcinoma. Interestingly, in these cases, GATA3 was retained in the adjacent precursor lesion of differentiated VIN as well. This finding indicates that a separate molecular mechanism, independent of GATA3, may be involved in a subset of vulvar squamous cell carcinomas. Given the overlapping immunohistochemistry patterns of GATA 3 in the majority of differentiated VIN and associated invasive squamous cell carcinomas in our study, we conclude that the loss of GATA3 expression cannot help distinguish between differentiated VIN with pseudo-invasion and differentiated VIN with true stromal invasion. Further, our observation that $19 \%$ of vulvar squamous cell carcinomas retained significant GATA3 immunoreactivity, also argues against its application in this diagnostic difficulty.

The role of GATA3 in HPV-induced carcinogenesis is largely unknown. Our data of retained GATA3 expression in all usual type VIN cases suggests that GATA3 is not directly involved, at least in the early stage of HPV-related carcinogenesis. To our surprise in our study, all seven cases of invasive squamous cell carcinoma associated with usual type VIN showed significant loss of GATA3 expression. Our results may imply that GATA3 downregulation occurs at a later stage in HPV-related vulvar squamous neoplasia as opposed to HPV-unrelated vulvar squamous neoplasia. This speculation is supported by a previous in vitro study of cervical keratinocytes. Steenbergen et al. [31] demonstrated that HPV-mediated immortalization of human keratinocyte cell lines in vitro was associated with a marked reduction in both GATA3 mRNA and protein expression. On immunohistochemistry, they showed that $67 \%$ of cervical squamous cell carcinomas exhibited complete absence of GATA3 staining in contrast to only $11 \%$ of cervical intraepithelial neoplasia 3 lesions. They concluded that complete downregulation of GATA3 represents a rather late event in cervical squamous carcinogenesis [31]. Solus et al. [32] have shown that there was a more significant loss of GATA3 immunostaining in premalignant squamous lesions of sun-exposed skin as compared to squamous cell carcinoma in situ of Bowenoid type arising in sun-protected skin, indicating differences in the early steps of actinic versus Bowenoid squamous transformation.

In summary, our study provides new insight into the pathways of development of the two types of VIN. 
Downregulation of GATA3 appears to be associated with both pathways of invasive vulvar squamous cell carcinoma. The progressive loss of GATA3 from differentiated VIN to invasive squamous cell carcinoma indicates that the downregulation of GATA3 occurs in the early stage of HPV-independent vulvar carcinogenesis. The pattern of GATA3 expression in differentiated VIN is distinct from that of the non-neoplastic epidermis and usual type VIN and can serve as a useful tool in facilitating its pathologic diagnosis.

\section{Compliance with ethical standards}

Conflict of interest The authors declare that they have no conflict of interest.

\section{References}

1. Wilkinson EJ. Premalignant and malignant tumors of the vulva. In: Kurman RJ, Ellenson LH, Ronnett BM, editors. Blaustein's pathology of the female genital tract. 6th ed. New York: Springer; 2011. p. 64

2. Hart WR. Vulvar intraepithelial neoplasia: historical aspects and current status. Int J Gynecol Pathol. 2001;20:16-30.

3. Del Pino M, Rodriguez-Carunchio L, Ordi J. Pathways of vulvar intraepithelial neoplasia and squamous cell carcinoma. Histopathology. 2013;62:161-75.

4. Reyes MC, Cooper K. An update on vulvar intraepithelial neoplasia: terminology and a practical approach to diagnosis. J Clin Pathol. 2014;67:290-4.

5. van de Nieuwenhof HP, Massuger LF, van der Avoort IA, et al. Vulvar squamous cell carcinoma development after diagnosis of VIN increases with age. Eur J Cancer. 2009;45:851-6.

6. Smith JS, Backes DM, Hoots BE, et al. Human papilloma virus type-distribution in vulvar and vaginal cancers and their associated precursors. Obstet Gynecol. 2009;113:917-24.

7. Léonard B, Kridelka F, Delbecque K, et al. A clinical and pathological overview of vulvar condyloma acuminatum, intraepithelial neoplasia, and squamous cell carcinoma. Biomed Res Int. 2014;2014:480573.

8. de Sanjosé S, Alemany L, Ordi J, et al. Worldwide human papilloma virus genotype attribution in over 2000 cases of intraepithelial and invasive lesions of the vulva. Eur J Cancer. 2013;49:3450-61.

9. Trietsch MD, Nooij LS, Gaarenstroom KN, et al. Genetic and epigenetic changes in vulvar squamous cell carcinoma and its precursor lesions: a review of the current literature. Gynecol Oncol. 2015;136:143-57.

10. Pinto AP, Miron A, Yassin Y, et al. Differentiated vulvar intraepithelial neoplasia contains Tp53 mutations and is genetically linked to vulvar squamous cell carcinoma. Mod Pathol. 2010;23:404-12.

11. Rolfe KJ, MacLean AB, Crow JC, et al. TP53 mutations in vulval lichen sclerosus adjacent to squamous cell carcinoma of the vulva. Br J Cancer. 2003;89:2249-53.

12. Choschzick M, Hantaredja W, Tennstedt P, et al. Role of TP53 mutations in vulvar carcinomas. Int $\mathrm{J}$ Gynecol Pathol. 2011;30:497-504.

13. Zheng R, Blobel GA. GATA transcription factors and cancer. Genes Cancer. 2010;1:1178-88.
14. Masse I, Barbollat-Boutrand L, Kharbili ME, et al. GATA3 inhibits proliferation and induces expression of both early and late differentiation markers in keratinocytes of the human epidermis. Arch Dermatol Res. 2014;306:201-8.

15. Miettinen M, Mc. Cue PA, Sarlomo-Rikala M, et al. Gata 3 - a multispecific but potentially useful marker in surgical pathology a systematic analysis of 2500 epithelial and non-epithelial tumors. Am J Surg Pathol. 2014;38:13-22.

16. Mertens RB, de Peralta-Venturina MN, Balzer BN, et al. GATA3 expression in normal skin and in benign and malignant epidermal and cutaneous adnexal neoplasms. Am J Dermatopathol. 2015;37:885-91.

17. Abell MR. Intraepithelial carcinomas of epidermis and squamous mucosa of vulva and perineum. Surg Clin North Am. 1965;45:1179-98.

18. van de Nieuwenhof HP, Bulten J, Hollema H, et al. Differentiated vulvar intraepithelial neoplasia is often found in lesions, previously diagnosed as lichen sclerosus, which have progressed to vulvar squamous cell carcinoma. Mod Pathol. 2011;24:297-305.

19. van den Einden LC, de Hullu JA, Massuger LF, et al. Interobserver variability and the effect of education in the histopathological diagnosis of differentiated vulvar intraepithelial neoplasia. Mod Pathol. 2013;26:874-80.

20. Yang B, Hart WR. Vulvar intraepithelial neoplasia of the simplex (differentiated) type: a clinicopathologic study including analysis of HPV and p53 expression. Am J Surg Pathol. 2000;24:429-41.

21. Liegl B, Regauer S. p53 immunostaining in lichen sclerosus is related to ischaemic stress and is not a marker of differentiated vulvar intraepithelial neoplasia (d-VIN). Histopathology. 2006;48:268-74.

22. Hantschmann P, Sterzer S, Jeschke U, et al. P53 expression in vulvar carcinoma, vulvar intraepithelial neoplasia, squamous cell hyperplasia and lichen sclerosus. Anticancer Res. 2005;25:1739-45.

23. Singh N, Leen SL, Han G, et al. Expanding the morphologic spectrum of differentiated VIN (dVIN) through detailed mapping of cases with p53 loss. Am J Surg Pathol. 2015;39:52-60.

24. McCleskey BC, Penedo TL, Zhang K, et al. GATA3 expression in advanced breast cancer: prognostic value and organ-specific relapse. Am J Clin Pathol. 2015;144:756-63.

25. Kouros-Mehr H, Bechis SK, Slorach EM, et al. GATA3 links tumor differentiation and dissemination in a luminal breast cancer model. Cancer Cell. 2008;13:141-52.

26. Yan W, Cao QJ, Arenas RB, et al. GATA3 inhibits breast cancer metastasis through the reversal of epithelial-mesenchymal transition. J Biol Chem. 2010;285:14042-51.

27. Chang A, Amin A, Gabrielson E, et al. Utility of GATA3 immunohistochemistry in differentiating urothelial carcinoma from prostate adenocarcinoma and squamous cell carcinomas of the uterine cervix, anus, and lung. Am J Surg Pathol. 2012;36:1472-6.

28. Clark BZ, Beriwal S, Dabbs DJ, et al. Semiquantitative GATA3 immunoreactivity in breast, bladder, gynecologic tract, and other cytokeratin 7-positive carcinomas. Am J Clin Pathol. 2014;142:64-71.

29. Bonin F, Molina M, Malet C, et al. GATA3 is a master regulator of the transcriptional response to low-dose ionizing radiation in human keratinocytes. BMC Genomics. 2009;10:417.

30. Nakayama A, Suzuki S, Hashimoto N, et al. Role of tumor suppressor p53, RB and GATA3 network in the regulation of malignant transformation of breast cancer cells via Srebpdependent cholesterol synthetic pathway. Poster presentation at the Endocrine Sosiety's 98th Annual Meeting and Expo, April1-4, 2016, Boston. Online access at https://www.press.endocrine.org/ doi/abs/10.1210/endo-meetings.2016.TB.1.FRI-060. 
31. Steenbergen RD, OudeEngberink VE, Kramer D, et al. Downregulation of GATA3 expression during human papilloma virusmediated immortalization and cervical carcinogenesis. Am J Pathol. 2002;160:1945-51.
32. Solus JF, Hassan K, Lee SJ, et al. Cutaneous squamous cell carcinoma progression is associated with decreased GATA3 immunohistochemical staining. J Cutan Pathol. 2016;43: $347-53$. 